\title{
Multi-Ensayos
}

Vol. 5, $\mathrm{N}^{\circ} 9$

ISSN: 2412-3285

https://multiensayos.unan.edu.ni

DOI: https://doi.org/10.5377/multiensayos.v5i9.9433

\section{Generando ideas de valor para el emprendimiento desde la asignatura de prácticas de familiarización}

\section{Generating ideas of value for entrepreneurship from the subject of familiarization practices}

Elimelec Josué Videa Merlo

\section{RESUMEN}

Recibido: 21 de enero de 2019, Aceptado: 17 de mayo de 2019

Las Prácticas de Familiarización desarrolladas durante el segundo semestre 2017 con estudiantes de tercer año de Mercadotecnia, han sido el escenario perfecto para trabajar desde una perspectiva práctica el concepto del emprendimiento y la innovación, debido a que es una asignatura que pretende aportar al desarrollo de las capacidades, habilidades y destrezas del estudiante, fomentando el pensamiento creativo como vinculo necesario para desarrollar el pensamiento empresarial. Dadas las condiciones temáticas, desde el principio de la clase se ha discutido la importancia de las prácticas, el rol del estudiante como generador de cambio y la necesidad imperante de generar emprendimiento como medida sostenible para el desarrollo y para transformar su realidad.

Palabras claves: prácticas de familiarización; emprendimiento.

\section{ABSTRACT}

Familiarization internship done during the second semester 2017 with third year marketing students, have been the perfect scenario to work from a practical perspective the concept of entrepreneurship and innovation, because it is a subject that aims to contribute to the development of the skills, abilities and expertise of the student, encouraging creative thinking as a necessary connection to develop business thinking. Due to the thematic conditions, since the beginning of the class has been discussed the importance of internship, the role of the student as a generator of change and the imperative to generate entrepreneurship as a sustainable technique to develop and transform their reality.

Keywords: familiarization practices; entrepreneurship.

1 Docente del departamento de Ciencias Económicas y Administrativas, UNAN-Managua/FAREM-Estelí. Correo electrónico: elimelec.videa@gmail.com.

(C) 2019 Revista Multi-Ensayos. 


\section{INTRODUCCIÓN}

Muchas personas se preguntan cuál es la mejor forma de emprender. Aquí inicia una de las aventuras que más satisfacen cuando salen bien las cosas, pero que más decepcionan en el supuesto de que las ideas no terminen calando. El proceso de emprendimiento incorpora cuestiones difíciles de responder, sobre todo cuando los temores y los riesgos suponen la mejor compañía de una idea exitosa.

El emprendimiento comienza antes que nada con la motivación, sin embargo aquí se discute el papel preponderante de la generación de ideas de valor, lo cual implica la solución de problemas, necesidades explicitas y latentes del consumidor de una manera innovadora. Esta ha sido la base para que los estudiantes plantearan durante la clase diferentes planes de negocios basados en ideas creativas adecuadas a su contexto y perfil profesional.

\section{DESARROLLO}

La temática del emprendimiento como eje transversal de la asignatura supone un reto y grandes esfuerzos de parte de todos los actores involucrados, rompiendo la inercia del aula y la dependencia profesor/alumno. El docente debe identificar las expectativas del alumno, guiar y construir un ambiente que propicie interés confianza y ganas de trabajar. Tiene que estructurar los proyectos, actividades y estudios que promuevan el emprendimiento, seleccionando escenarios, herramientas y métodos más convenientes.

La mejor forma de emprender será siempre un cuestionamiento con muchas opciones de respuesta, si consideramos las diferentes maneras de hacerlo, sin embargo; como resultado de la experiencia trabajada con los estudiantes, puede definirse en dos simples pero complejas palabras: actitud y estímulo; no es posible generar emprendimiento sin tener una actitud hacia el mismo, motivaciones y metas de superación, pero ello debe estar acompañado de estímulos que son en principio, inherentes al ser humano y otros que deben ser desarrollados, en este aspecto podemos hacer referencia a las competencias de cada individuo, a su nivel de conocimiento, sus experiencias y su capacidad de generar ideas ganadoras.

Para lograr el efecto del pensamiento emprendedor se trabajó con una metodología práctica, participativa y centrada en las necesidades del estudiante, lo cual implicó para el docente tener en cuenta factores críticos como la formación alcanzada hasta esa fase de su carrera, y la aplicación de técnicas para rescatar sus aptitudes, centrándose en el conocimiento y la creatividad. Este proceso requiere del desarrollo de habilidades y el estímulo de diferentes tipos de pensamientos (entre ellos el pensamiento divergente) y la inteligencia, elementos que permiten generar ideas creativas mediante la exploración de muchas posibles soluciones a los problemas del entorno, pero además requiere cambiar muchos paradigmas que atan la manera de pensar del emprendedor.

Durante el desarrollo de la asignatura se trabajaron herramientas como el brainstorming (Lluvia de ideas), el modelo Canvas, aplicaciones de branding (creación de marcas), técnicas de ventas para la presentación de ideas, así como el uso de una estructura de plan de negocios práctica, propiciando un ambiente creativo que llevó al desarrollo de diferentes trabajos, entre los cuales se mencionan algunos:

Homex: Una iniciativa cuyo slogan es "lo hacemos más fácil", centrada en dar respuesta a usuarios de servicios domésticos y domiciliares, como el cuidado de menores, reparaciones domésticas, transporte local, basado en una propuesta de valor centrada en la logística y el servicio a tiempo las 24 horas del día. 
Green Food: Un restaurante cuyo slogan es "delicioso y saludable" con un concepto diferenciado por la oferta de productos en la categoría de comida sana, dirigido a personas con una educación y conducta orientada a un estilo de vida saludable.

Innovate (Asesoría y Marketing): Una iniciativa desarrollada para dar respuesta a las Mipymes del norte de Nicaragua, considerando la necesidad latente de servicios profesionales de mercadotecnia a un costo accesible y ajustado a los requerimientos específicos del cliente.

Multi-eventos: Una iniciativa empresarial que responde a la necesidad insatisfecha de un mercado que demanda la existencia de una empresa dedicada a la organización, asesoría y montaje de eventos sociales y empresariales con calidad.

El Diamante (Deportes Extremos): Una empresa dirigida al campo del entretenimiento extremo, enfocada en un segmento joven con un estilo de vida aventurera y dispuesta a vivir los mejores retos del deporte; lo cual responde a la necesidad en el mercado de un centro de entretenimiento con características diferenciadoras y fuera de lo común.

\section{CONCLUSIÓN}

Es importante destacar la visión novedosa del estudiante y la manera en que materializa sus ideas, de los cual se rescatan dos aspectos interesantes; a) la combinación de diferentes áreas de interés y su vinculación con las necesidades del mercado y el perfil profesional, b) la construcción de un prototipo del modelo de negocios basado en la creatividad y la innovación, trayendo como resultado una actitud emprendedora que seguramente causará efectos en el mediano y largo plazo.

La promoción del emprendimiento es una prioridad dentro del proceso de formación, puesto que modificando la tendencia hacia la búsqueda de empleo y aumentando las iniciativas empresariales de forma innovadora, se puede lograr la creación de más y mejores empresas. La idea clave es contribuir a que el estudiante vea en el emprendimiento una alternativa para trabajar dignamente y tener una buena calidad de vida. Una sociedad requiere de emprendedores, con iniciativa, innovadores, líderes que trabajen para construir su futuro, y una manera de lograrlo es desde la educación.

\section{REFERENCIAS}

Ministerio de Industria, Comercio, Minería y Desarrollo Científico Tecnoñógico del gobierno de Córdoba. (2014). Emprendiendo: Herramientas para emprendedores en formación. Córdoba, Argentina.

Osterwalder, A., \& Pigneur, Y. (2010). Generación de modelos de negocio: Un manual para visionarios, revolucionarios y retadores. Barcelona, España: Centro Libros PAPF.

Rubio, G., López, L., \& Parra, M. (2017). Emprendimiento y creatividad: Aspectos esenciales para crear empresa. Colombia: ECOE.

UNAN-Managua. (2015). Programa de Asignatura: Prácticas de Familiarización. Managua.

Valls, J., Cruz, C., Torruella, A., Juanes, E., Canessa, M., \& Hormiga, E. (2012). Causas de fracaso de los emprendedores. La Coruña, España: Netbiblo, S.L. 


\section{ANEXOS}

Marcas de proyectos desarrollados
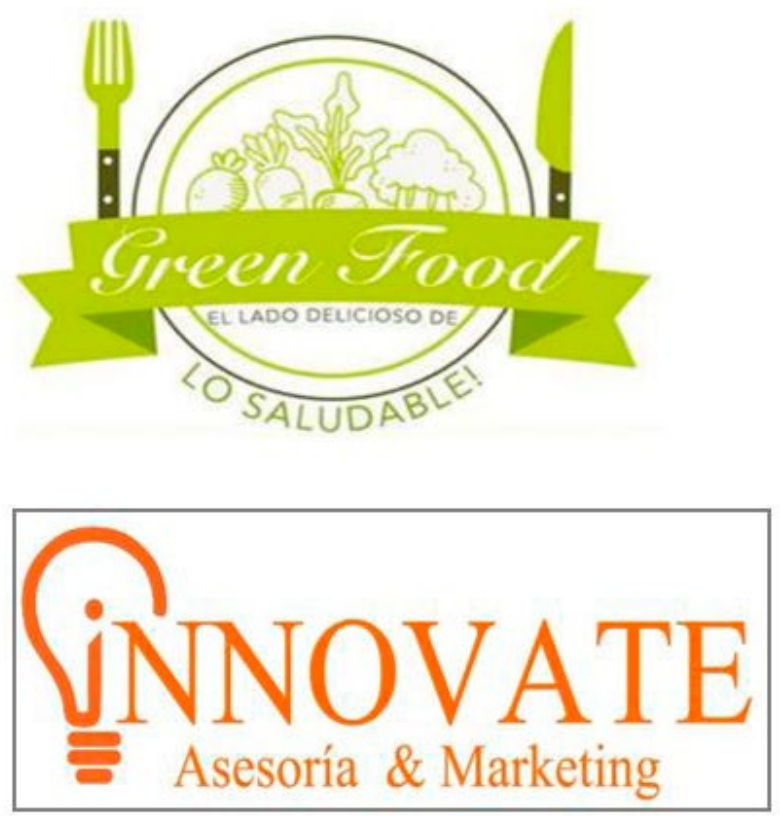
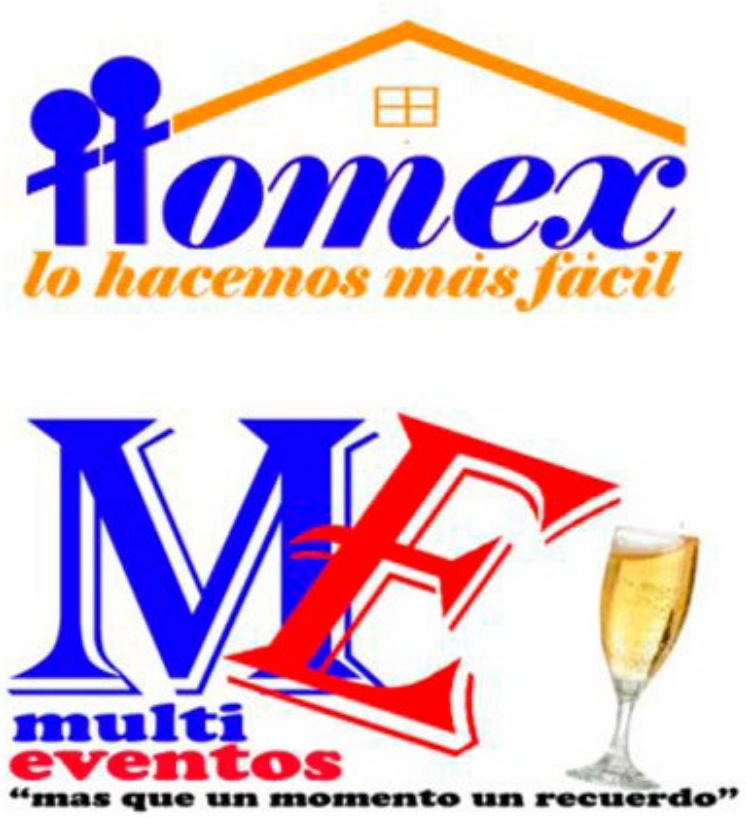